\title{
A One-Step Immunoassay for Carbohydrate Antigen 19-9 by Biosensor Based on Imaging Ellipsometry
}

\author{
Chenghong Huang, ${ }^{1,2,4}$ Yanyan Chen, ${ }^{1}$ and Gang JiN ${ }^{1,2,3}$ \\ ${ }^{1}$ Suzhou Institute of Nano-tech and Nano-bionics, Chinese Academy of Sciences, Suzhou 215123, China; ${ }^{2}$ Institute of \\ Biophysics, Chinese Academy of Sciences, Beijing 100101, China; ${ }^{3}$ Key Laboratory of Microgravity, Institute of Mechanics, \\ CAS, Beijing 100190, China; and ${ }^{4}$ Graduate School of the Chinese Academy of Sciences, Beijing, China
}

(Received 7 June 2010; accepted 21 September 2010; published online 30 September 2010)

Associate Editor Tingrui Pan oversaw the review of this article.

\begin{abstract}
Cancer markers can offer much more information for developing or developed cancers. Simple and shorttime assay of cancer biomarker is essential for clinic diagnosis and management. In this study, a one-step immunoassay for carbohydrate antigen 19-9 (CA19-9) by biosensor based on imaging ellipsometry (BIE) is described and its analysis performances are evaluated. Anti-CA19-9 monoclonal antibody as ligand covalently immobilized on carboxyl-modified silicon substrate and its immobilization concentration concurrently screened. Then, bovine serum albumin (BSA) is used to block for acquisition of sensing layer. The detection linear range is from 15.0 to $120.0 \mathrm{U} / \mathrm{mL}$ with a minimum limit of $10.0 \mathrm{U} / \mathrm{mL}(\mathrm{S} / \mathrm{N}=3)$. The intraslide and inter-slide reproducibility, expressed as coefficient variation $(\mathrm{CV} \%)$, are $9.9,5.9,5.4 \%$ and $9.6,7.6,5.3 \%$ for $31.9,57.4$, and $99.2 \mathrm{U} / \mathrm{mL}$ of CA19-9, respectively. Accuracy determined by spiked-recovery is among $95.1-106.7 \%$. Specificity expressed as cross-reaction rate are all below $5.8 \%$ evaluated by three analogous biomarkers alphafetoprotein (AFP), carcinoembryonic antigen (CEA), and carbohydrate antigen 242 (CA242). Application of BIE to 25 clinic samples demonstrated that the results are in high correlate with electrochemiluminescent immunoassay (ECLIA, $\left.R^{2}=0.997\right)$. The proposed immunoassay has the potential for clinic application.
\end{abstract}

Keywords-Biosensor, Imaging ellipsometry, Carbohydrate antigen 19-9, Immunoassay.

\section{INTRODUCTION}

Biomarkers can offer much more information for identification of early cancer and people at risk of developing cancer, ${ }^{17}$ therefore, their sensitive detection and accurate analysis are very essential for cancer

\footnotetext{
Address correspondence to Yanyan Chen and Gang Jin, Suzhou Institute of Nano-tech and Nano-bionics, Chinese Academy of Sciences, Suzhou 215123, China. Electronic mail: huang1ng@tom.com, yychen2006@sinano.ac.cn, gajin@imech.ac.cn
}

diagnosis and management. Carbohydrate antigen 19-9 (CA19-9) is always associated with pancreatic, gastric, and other gastrointestinal tract cancers. $6,7,26$ The measurement of CA19-9 in clinic mainly depends on conventional electrochemiluminescent immunoassay (ECLIA) $)^{14,34}$ at present. Although possessing high sensitivity and broader detection range, this technique, however, has some disadvantages including multiple reaction steps and time-consuming. Biosensor based on imaging ellipsometry (BIE), as a typical label-free and non-destructive technique for thin layer analysis, ${ }^{32}$ is high sensitive for film thickness to subnanometer resolution. ${ }^{37}$ It has been greatly advanced since the concept was first put forward by Jin. ${ }^{9,10}$ Previous work theoretically and empirically verifies that it can be used in many fields of bioassay including specific combination of antigen-antibody, virus examination, and biomarker measurement. ${ }^{19-22,31,38}$ In this study, a one-step immunoassay for CA19-9 utilizing BIE is demonstrated. The results are also compared with ECLIA by correlation analysis. The future application of BIE is also discussed.

\section{MATERIALS AND METHODS}

\section{Materials}

Silicon wafers are purchased from General Research Institute for Nonferrous Metals of China. Human CA19-9 and anti-human CA19-9 monoclonal antibody (Lot C081001, Clone 1116-NS-19-9) are purchased from Shanghai Linc-Bio Science Co. Bovine serum albumin (BSA) is obtained from Beijing Dingguo Co. 3-Aminopropyltriethoxysilane (APTES, 99\%), 1-(3-Dimethylaminopropyl)-3-ethylcarbobiimide hydrochloride (EDC), $\mathrm{N}$-hydroxysuccinimide (NHS), and 
succinimide anhydride are obtained from ACROS. Tween-20 is purchased from Sigma-Aldrich. Deionized water (resistivity $18.3 \mathrm{M} \Omega \mathrm{cm}$ ) is produced by ion exchange demineralization, followed by passing through a Milli-Q plus system from Millipore (Millipore, Bedford, MA).

\section{ECLIA Measurement of CA19-9}

The measurement of CA19-9 was carried out using CA19-9 immunoassay Kits (Roche Elecsys 2010). All operations are performed in the light of the specifications and in the division of Shandong Medical Institute Radioimmunoassay Laboratory, Department of Shandong Medical Academy. According to the calibration curve, the range of CA19-9 in collected serum samples is $0.89-1027.0 \mathrm{U} / \mathrm{mL}$.

\section{BIE Measurement of CA19-9}

\section{BIE Platform}

$\mathrm{BIE}^{9}$ has been developed in our laboratory for the purpose of performing parallel immunoassay and is based on a combination of imaging ellipsometry (IE) and microfluidic array system. IE (Fig. 1a) is used for reading the protein arrays. The microfluidic array system (Fig. 1b) is used for ligand immobilization, surface patterning, array fabrication, solution delivery, and antigen capturing.

IE is an enhancement of conventional ellipsometry, which equips with a CCD camera to image the ellipsometry response of a larger area on the sample surface. ${ }^{11}$ The light source is a Xenon lamp, and a specific collimating system is used to provide an expanded parallel probing beam with a diameter of about $25 \mathrm{~mm}$. The beam passes through a polarizer and a compensator and finally on the sample surface at an incident of $75^{\circ}$. An optical filter at $632.8 \mathrm{~nm}$ wavelength is placed in the incident optical passage. The reflected beam containing sample information passes through an analyzer and an imaging lens and finally arrives to CCD camera. A digital image was grabbed by and stored in a computer with a grayscale format (8 bits, 0-255 grayscale) for further analysis. If the refractive index of sample layer is invariant, the grayscale value will be a function of the thickness of the sample layer, i.e., $I=f(d)$, where $I$ is the light intensity,
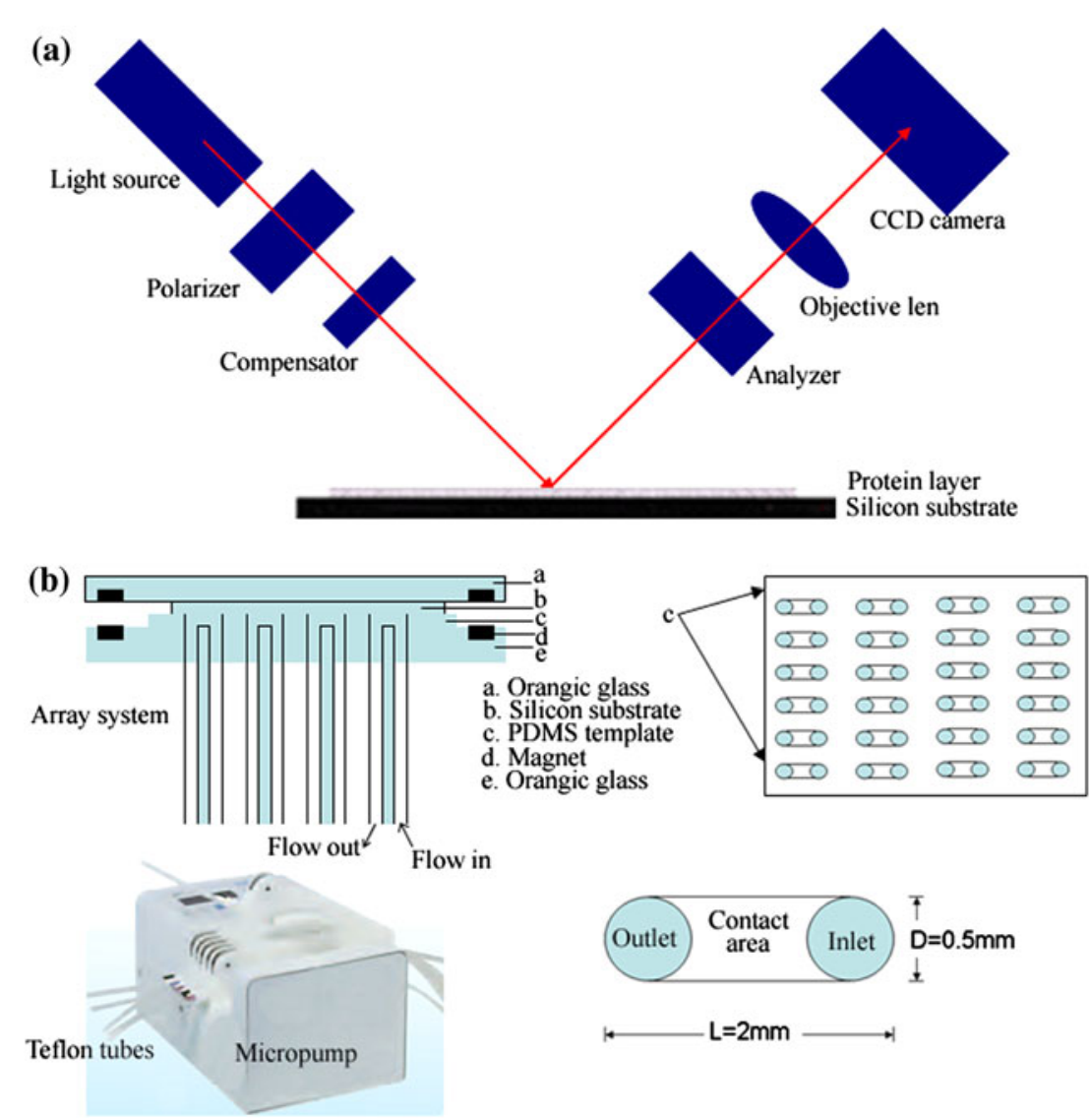

FIGURE 1. Schematic diagram of the biosensor based on imaging ellipsometry (BIE) system. (a) Illustration of imaging ellipsometry and (b) microfluidic array system. 
$f()$ is the function relationship, and $d$ is the layer thickness. Under these conditions, $f()$ can be determined from protein layers with known grayscale values and known thicknesses ${ }^{1}$ At the same time, there is a relationship between surface concentration and film thickness: surface concentration $\left(\mu \mathrm{g} / \mathrm{cm}^{2}\right) \approx K \times d$, where $K=0.12 .{ }^{18}$ Thus, the grayscale value directly reflects layer thickness and surface concentration. The higher the grayscale value, the thicker the layer and the higher the surface concentration.

The microfluidic array system is used for surface patterning and array production. It has three main parts including multi-cell array, microchannels, and micropump. Multi-cell array on polydimethylsiloxane (PDMS) template is used for array fabrication. ${ }^{27}$ Each cell has an inlet and an outlet. Both of them are connected by Teflon tubes for formation of microchannel. The inlet is put into sample plate for sample sucking and outlet is connected with micropump (ISMATEC, Switzerland) offering negative pressure so that sample can be flow in and flow out through the cell. ${ }^{29}$ Protein solutions are delivered to different cells using microfluidic system so that the surface is patterned homogeneously and simultaneously in array format. The simple channel junctions can be used in serial or parallel formats to analyze single or multiple samples simultaneously. ${ }^{8}$

\section{Silicon Wafer Modification}

Silicon wafers were cut into $2 \times 1 \mathrm{~cm}$ rectangles and rinsed with deionized water. The cleaned silicon wafers were initially treated with piranha solution $(30 \%$ $\mathrm{H}_{2} \mathrm{O}_{2}: 98 \% \mathrm{H}_{2} \mathrm{SO}_{4}=1: 3, \mathrm{v} / \mathrm{v}$ ) for $30 \mathrm{~min}$. This step made the surface carrying with hydrophilic $-\mathrm{OH}$ groups. After washed by deionized water, silicon wafers were immersed in a mixture of APTES and absolute ethanol (APTES: absolute ethanol = 1:10, v/v) and incubated for $2 \mathrm{~h}$. The reaction of APTES with the surface resulted in covalent immobilization of $-\mathrm{O}-\mathrm{Si}-$ $\left(\mathrm{CH}_{2}\right)_{3}-\mathrm{NH}_{2}$, forming a layer of densely packed amino groups on the surface. ${ }^{28}$ After washed by absolute ethanol, the wafers were incubated in a saturated succinic anhydride solution in ethanol for $12 \mathrm{~h}$. The $\mathrm{CH}_{2} \mathrm{CH}_{2} \mathrm{COOCO}$ of succinic anhydride reacts with $-\mathrm{O}-\mathrm{Si}-\left(\mathrm{CH}_{2}\right)_{3}-\mathrm{NH}_{2}$ immobilized on the surface, generating $-\left(\mathrm{CH}_{2}\right)_{3} \mathrm{NH}-\mathrm{CO}\left(\mathrm{CH}_{2}\right)_{2}-\mathrm{COOH}^{2}$ The modified wafers were stored in ethanol solution at $4{ }^{\circ} \mathrm{C}$ until use.

\section{Ligand Immobilization and Concentration Screening}

Rat anti-human CA19-9 monoclonal antibody was used as ligand. When the modified silicon wafer was impressed on PDMS template, carboxyl groups on its surface was first activated by solution of 1-(3-dimethylaminopropyl)-3-ethylcarbodiimide hydrochloride (EDC/0.2 M) $/ N$-hydroxysuccinimide (NHS/0.05 M), and $25 \mu \mathrm{L}$ of EDC/NHS solution was then added to the microfluidic system at $5 \mu \mathrm{L} / \mathrm{min}$ and passed through each cell. In the presence of NHS, EDC could transfer carboxyl groups to the Sulfo-NHS ester which could then react with the amino groups of the protein. ${ }^{36}$ Subsequently, the silicon wafer was treated with anti-CA19-9 monoclonal antibody as ligand in PBS at $1 \mu \mathrm{L} / \mathrm{min}$ at room temperature for $30 \mathrm{~min}$ using microfluidic system. These ligand layers were further blocked by BSA solution $(1 \mathrm{mg} / \mathrm{mL})$ at $1 \mu \mathrm{L} / \mathrm{min}$ for $30 \mathrm{~min}$ and then can be used for antigen binding analysis. In order to screen the concentration of ligand that would produce the most optimal antigen binding capacity, different concentrations of CA19-9 in PBS were added to perform matrix testing. After the assay completed, silicon is taken to IE measurement.

\section{Establishment of Standard Curve}

Total 10 cell units were used for establishment of standard curve. Eight of them were used for serial diluted positive sample containing antigen CA19-9 of $413.8,206.9,103.5,51.7,25.8,12.9,6.45$, and $0.0 \mathrm{U} / \mathrm{mL}$, respectively. The other two were served as positive control of standard $(37.0 \mathrm{U} / \mathrm{mL}$, which is the cut-off value to distinguish the normal or abnormal of serum content $\left.{ }^{16}\right)$. Prepared antigen solutions were independently dispensed to the unit dots to react for 30 min with a flow of $1 \mu \mathrm{L} / \mathrm{min}$ at room temperature for antigen capturing. After the antigen-antibody combination completed, all the unit were washed by PBST $(0.02 \%$ tween-20) and deionized water repeatedly with the flow rate of $20 \mu \mathrm{L} / \mathrm{min}$ for $5 \mathrm{~min}$, then, rendered to IE for grayscale measurement.

\section{Sensitivity, Reproducibility, Accuracy, and Specificity}

The minimum detectable limit of the assay was determined by adding three standard deviations to the mean grayscale value of 10 zero standard replicates and calculating the corresponding concentration, when interpolated from the standard curve. Reproducibility was determined by calculating the intra-slide and interslide coefficient variation $(\mathrm{CV} \%)$. The intra-slide reproducibility was obtained by calculating the intraslide of eight measurements of sera with in the same silicon substrate. The inter-slide reproducibility was obtained from six samples of sera in six consecutive assays with different silicon substrate. Accuracy of the assay was determined by recovery. To determine recovery of CA19-9 from serum sample, CA19-9 was spiked into serum of known concentrations at $31.9,57.4$, and $99.2 \mathrm{U} / \mathrm{mL}$. Specificity was assessed by 
cross-reaction rate with calculation of signal response percentage, which was performed with three analogous markers alpha-fetoprotein (AFP), carcinoembryonic antigen (CEA), carbohydrate antigen 242 (CA242) and their mixture.

\section{Real Serum Measurement}

Serum samples were collected from 25 patients with gastric cancer admitted to hospital. Serum samples were stored at $-70{ }^{\circ} \mathrm{C}$ until the time of analysis. Briefly, real samples or appropriate diluted samples were added to sensing layer to react $30 \mathrm{~min}(1 \mu \mathrm{L} / \mathrm{min})$ at room temperature. Each sample was at least measured twice and the mean value was accordingly calculated by the calibration curve made in a same substrate concurrently.

\section{RESULTS}

\section{Optimization of the Analytical Conditions}

\section{Screening of Ligand Concentration}

Ligand assembled on chemistry surface with excess concentration was unfavorable for antigen accessible due to their overlapping aggregation to form steric hindrance. ${ }^{30,35}$ On the other hand, ligand immobilized on chemistry surface with less concentration was also not helpful antigen combination as scarce of antigen binding sites. Thus, it is important to systematically screen the ligand concentration for biosensor development. The ellipsometric image is illustrated in Fig. 2. We dispensed different ligand with 40.0, 80.0, $120.0,160.0,200.0$, and $240.0 \mu \mathrm{g} / \mathrm{mL}$, respectively, to the chemistry surface and arranged vertically. Then, antigen CA19-9 with $0.0,1.7,5.1,15.3,46.0$, and $138.0 \mathrm{U} / \mathrm{mL}$ were separately delivered to react with the immobilized ligands horizontally. The biggest grayscale change $(\Delta G)$ induced by antigens combination was obtained when the concentration of $120 \mu \mathrm{g} / \mathrm{mL}$ ligand was used (Fig. 3) according to calculation of three dependent measurements, therefore, this concentration was chosen for fabrication of sensing layer.

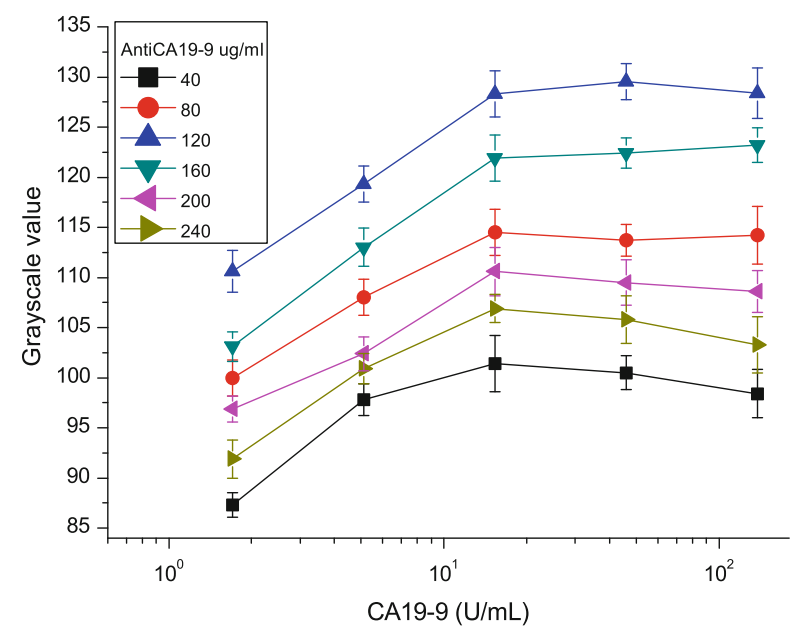

FIGURE 3. Grayscale value vs. anti-CA19-9 concentration was plotted for ligand concentration screening.

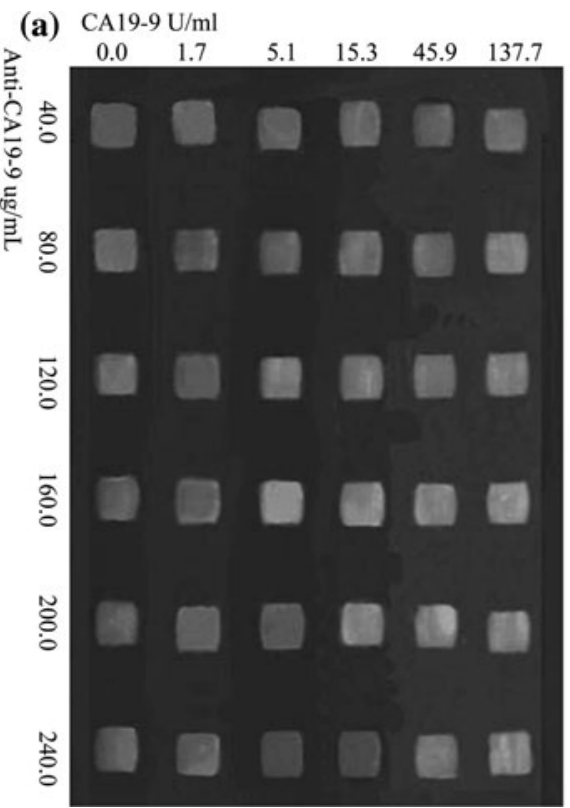

(b)

\begin{tabular}{|c|c|c|c|c|c|}
\hline 82.1 & 82.3 & 82.3 & 82.5 & 82.2 & 82.4 \\
\hline 87.3 & 100.0 & 110.6 & 112.4 & 96.9 & 115.3 \\
\hline 97.8 & 108.0 & 119.3 & 113.0 & 102.4 & 100.9 \\
\hline 101.4 & 114.5 & 128.3 & 121.9 & 110.6 & 106.9 \\
\hline 100.5 & 113.7 & 129.5 & 122.4 & 109.5 & 105.8 \\
\hline 98.4 & 114.2 & 128.4 & 123.2 & 108.6 & 103.3 \\
\hline
\end{tabular}

FIGURE 2. Ligand concentrations screened in matrix format. Serial diluted ligand solutions $(40.0-240.0 \mu \mathrm{g} / \mathrm{mL})$ are first immobilized on the chemistry substrate, and then their antigen binding activities are testified with corresponding antigen of CA19-9 (1.7-138.0 U/mL). The maximum grayscale value change is served as judgment for optimal ligand immobilization. 


\section{Optimization of Reaction Time}

The antigen reaction time was also optimized. After the sensing layer acquired, we independently carried out $10,20,30,40,50$, and $60 \mathrm{~min}$ antigen reaction with $100.0 \mathrm{U} / \mathrm{mL}$ CA19-9. The grayscale values with different reaction time were separately recorded. It revealed that equilibrium reaction achieved at $30 \mathrm{~min}$ approximately. Hence, this was chosen as antigen reaction time in next work.

\section{Optimization of Parameters Setup}

After the incident angle, wavelength and the dynamic range of CCD were fixed, the sensitivity of IE for CA19-9 analysis mainly depend on the azimuth angle parameters of polarizer, compensator, and analyzer that has been thoroughly discussed, ${ }^{4}$ which can significantly affect analysis performance of CA19-9. Here, when the azimuth angles of polarizer, compensator, and analyzer were fixed at $104^{\circ}, 45^{\circ}$, and $179^{\circ}$, respectively, the optimal sensitivity and acceptable detection range of CA19-9 were achieved.

\section{Sensitivity and Calibration Curve}

Total 10 analytical units were used to establish calibration curve. Seven units were served as serial diluted CA19-9 reaction and one for blank control (without CA19-9 addition). The other two were served as positive control. As shown in Fig. 4, the grayscale value for blank control is $88.2 \pm 1.3$ and $114.5 \pm 0.9$ for positive control $(37 \mathrm{U} / \mathrm{mL})$, respectively, suggesting that the sensitivity was enough and the judge for

(a)

(b) (c)

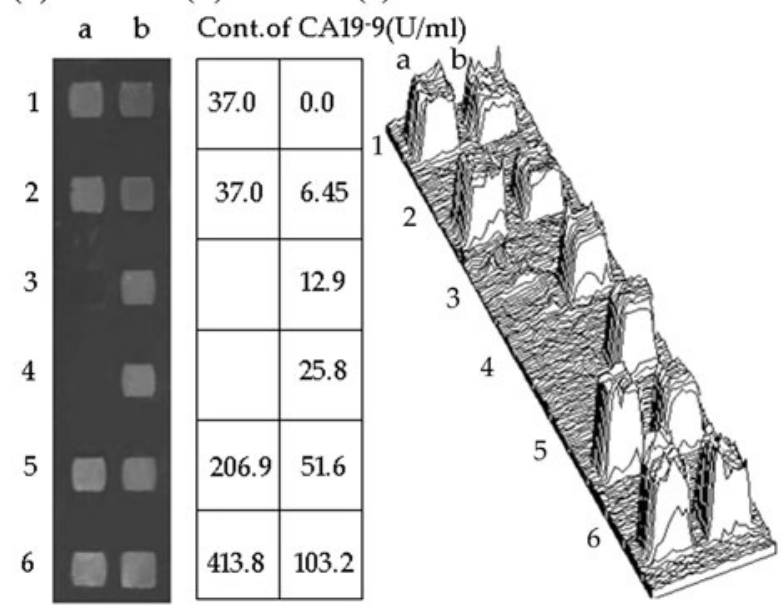

FIGURE 4. Typical dose-response ellipsometric image prepared with serial diluted CA19-9 in serum (413.8 U/mL). (a) Grayscale image, (b) corresponding CA19-9 content, and (c) three-dimension map. Unit a1 and a2 are served as positive control. b1 is a blank control, b2-6 and a5-6 are reference concentrations of CA19-9. normal or abnormal was effective. The minimum detection limit for detecting CA19-9 reached $10 \mathrm{U} / \mathrm{mL}$ $(\mathrm{S} / \mathrm{N}=3)$. Figure 4 shows that protein units turned brighter with increasing CA19-9 concentrations. The grayscale value directly reflects layer thickness and surface concentration, the thicker the layer and the higher the surface concentration. A calibration curve was obtained by four-parameter logistic function fitting based on the curve of the grayscale value versus the concentration of CA19-9 (Fig. 5) for three independent measurements. The curve could be represented by the regression equation: $y=29.5+$ $51.1 \log x \quad\left(R^{2}=0.98, \quad p<0.05\right)$ after logarithmic transformation, where $y$ is the grayscale value and $x$ is the CA19-9 concentration. The linear range was from 15.0 to $120.0 \mathrm{U} / \mathrm{mL}$.

\section{Specificity}

Antibody covalently immobilized on chemistry surface will partially inactivate resulting in loss of bioaffinity to some extent. ${ }^{12,24}$ The inactivation of antibody will lead to mismatch with non-target antigen introducing false positive results as multiple-related biomarkers will be produced during cancer developing. ${ }^{3,33}$ Thus, it is essential to evaluate the cross-reaction rate of the sensing layer. We calculated the percentage of grayscale response with three similar biomarkers AFP $(100.0 \mathrm{ng} / \mathrm{mL})$, CEA $(32.0 \mathrm{ng} / \mathrm{mL})$, CA242 $(100.0 \mathrm{U} / \mathrm{mL})$ and their mixture, which were all below $5.8 \%$ compared with $100.0 \mathrm{U} / \mathrm{mL}$ CA19-9 (Fig. 6). This validated that the proposed method has acceptable specificity.

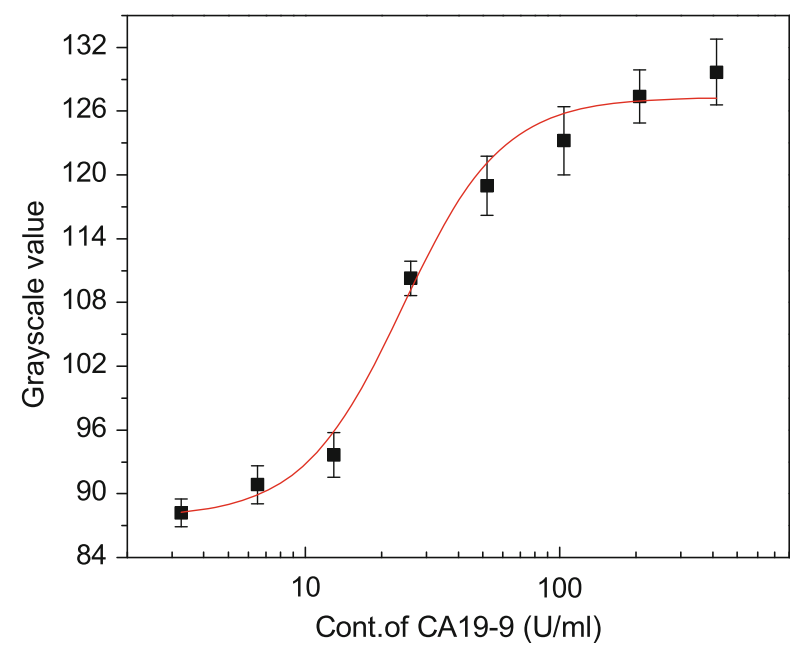

FIGURE 5. Calibration curve of the grayscale response vs. concentration of CA19-9 with the proposed immunoassay under optimal condition. Data are representative of three independent experiments. 


\section{Reproducibility}

The reproducibility was assessed by intra-slide and inter-slide measurements at low, medium, and high CA19-9 concentrations. The intra-slide reproducibility was tested with eight measurements in the same silicon wafer for the same serum concentration according to the guideline for protein chip evaluation. ${ }^{23}$ These series of analyses were repeated for three consecutive days in order to estimate the between-assay reproducibility. The results, expressed as mean $\mathrm{CV} \%$ for the three control sera, were shown in Table 1 . The $\mathrm{CV} \%$ values of intra-slide and inter-slide were 9.9, 5.9, 5.4\% and $9.6,7.6,5.3 \%$ for $31.9,57.4$, and $99.2 \mathrm{U} / \mathrm{mL}$ of CA19-9, respectively.

\section{Accuracy}

In order to further testify the accuracy of CA19-9 measurement, spiked-recovery rate profile was investigated. We employed a pool of sera at low, middle, and high CA19-9 concentration that all are within the detection range of BIE analysis to appraise the recovery rate. Table 2 reported the data for CA19-9 added

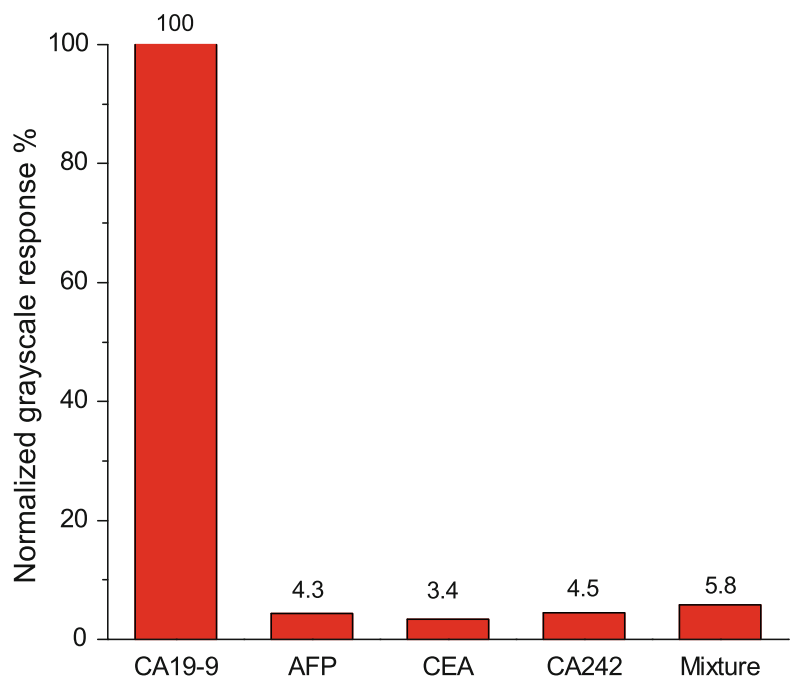

FIGURE 6. Specificity of BIE in the presence of CA19-9 (100.0 U/mL), AFP (100.0 ng/mL), CEA (32.0 ng/mL), CA 242 $(100.0 \mathrm{U} / \mathrm{mL})$, and their mixtures. and CA19-9 found. The spiked-recovery range is between 95.1 and $106.7 \%$, which has acceptable accuracy according to validation of bioassay in industry perspective. ${ }^{5}$

\section{Preliminary Application of BIE}

The BIE system was compared with a commercial ECLIA method (which is generally regarded as gold standard for biomarker measurement ${ }^{25}$ ) for the quantitative assay of CA19-9 by clinic samples. All of the sera were collected from gastric patients characterized by conventional pathology diagnosis. When the CA19-9 concentration exceeded the effective range of BIE analysis, it was diluted advisably by PBS. The assay procedure strictly adhered to the protocol established as above-mentioned. Each serum was tested twice and the mean value then was accordingly calculated after converting the grayscale into mass concentration. Total 25 samples were tested with the two methods and correlated. An illustration of typical ellipsometric image obtained by clinic sera was shown in Fig. 7. A high degree of correlation $(R=0.997$, $p<0.05$, Fig. 8) was obtained in various concentrations of samples by two methods.

\section{DISCUSSION AND CONCLUSION}

Compared with ECLIA, detection range of BIE is relatively narrow. This will not affect the CA19-9 measurement which can be solved by dilution of sample. The sensitivity is enough to distinguish normal and abnormal content because the detection limit of

TABLE 2. CA19-9 added and detected in the recovery tests.

\begin{tabular}{lccc}
\hline $\begin{array}{l}\text { Serum sample } \\
(\mathrm{U} / \mathrm{mL})\end{array}$ & $\begin{array}{c}\text { Spiked } \\
(\mathrm{U})\end{array}$ & $\begin{array}{c}\text { Found } \\
(\mathrm{U})\end{array}$ & $\begin{array}{c}\text { Recovery } \\
(\%)\end{array}$ \\
\hline 5.7 & 5.0 & 12.1 & 103.7 \\
5.7 & 10.0 & 16.4 & 104.5 \\
5.7 & 20.0 & 24.6 & 95.1 \\
5.7 & 40.0 & 48.2 & 106.7 \\
5.7 & 80.0 & 90.1 & 105.1 \\
\hline
\end{tabular}

TABLE 1. The intra-slide and inter-slide reproducibility (coefficient variation, \%) of BIE for CA19-9 measurement of three clinic samples.

\begin{tabular}{lcccccc}
\hline & \multicolumn{3}{c}{ Intra-slide reproducibility } & \multicolumn{3}{c}{ Inter-slide reproducibility } \\
\hline Sample & 1 & 2 & 3 & 1 & 2 & 3 \\
Number & 8 & 8 & 8 & 6 & 6 & 6 \\
Mean concentration $(\mathrm{U} / \mathrm{mL})$ & 31.9 & 57.8 & 99.4 & 29.16 & 59.42 & 102.13 \\
Standard deviation & 3.17 & 3.46 & 5.38 & 2.81 & 4.53 & 5.39 \\
CV (\%) & 9.9 & 5.9 & 5.4 & 9.6 & 7.6 & 5.3 \\
\hline
\end{tabular}


(a)

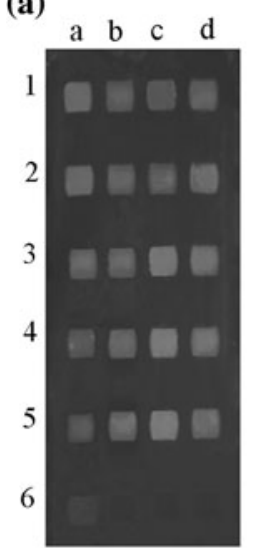

(b)

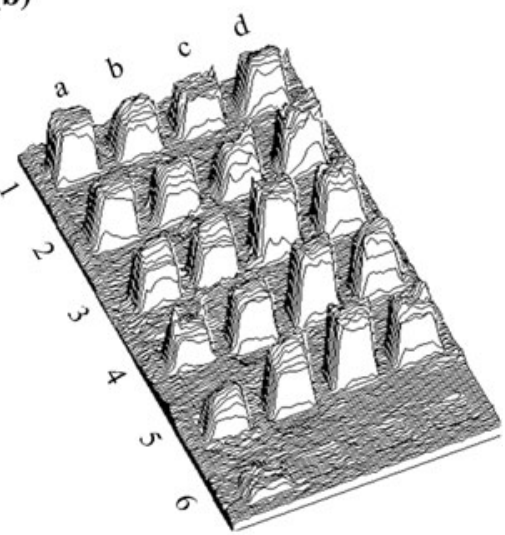

FIGURE 7. Clinical samples were measured by BIE with grayscale image (a) and corresponding three-dimension image (b). Units a1-a6 are reference concentration 100.0, 80.0, $60.0,40.0,20.0$, and $0.0 \mathrm{U} / \mathrm{mL}$ of CA19-9, which are served as establishment of calibration curve. b1-d5 are different content of clinic samples.

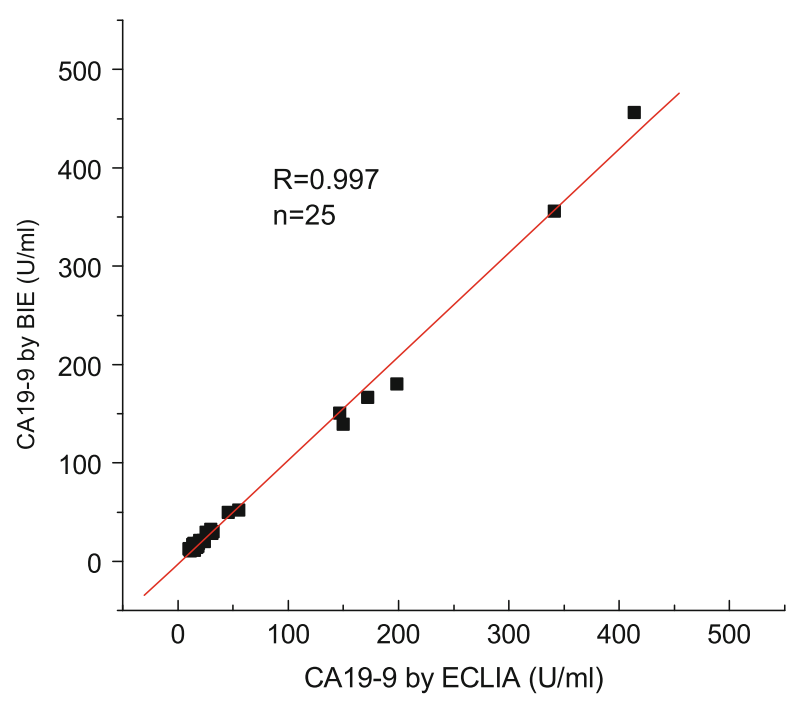

FIGURE 8. The correlation analysis of CA19-9 in patient serum by conventional ECLIA and proposed BIE analysis.

$10.0 \mathrm{U} / \mathrm{mL}$ is enough for CA19-9 assay which is under the cut-off value. In addition to the proposed immunoassay has advantages as follows: first, it is a typical label-free detection technique. It is reported that labeling of proteins can greatly change their surface characteristics, which can result in significant protein denature, especially for small proteins and peptide hormones. ${ }^{13}$ Therefore, BIE avoided disturbances from conjugated markers or handling with radioactive materials and largely simplified detection procedure, avoiding false positive results at most. Second, antibody can be patterned homogeneously and simultaneously on a same substrate in array format by the micro-fluidic system application. This is propitious to excellent reproducibility of BIE. ${ }^{27}$ Third, samples collected in hospitals are usually detected all at once. With simple micro-fluidic channel junction by Teflon tubes, the protein microfluidic array can be used in serial or parallel formats to analyze single or multiple samples simultaneously for high throughput. Fourth, the immunoassay still has advantages as sample delivery automation, less sample consuming (only $30 \mu \mathrm{L}$ sample is required for a single test) and short time (only $30 \mathrm{~min}$ is needed). Finally, BIE system is progressing in miniaturization and already obtained agitating fruit. ${ }^{15}$ Thus, the proposed immunoassay possesses considerable practical value.

To summary, we demonstrated here a promising one-step immunoassay for CA19-9 in serum. By taking advantage of comprehensive capability of BIE, antiCA19-9 monoclonal antibody as ligand can be covalently immobilized on carboxyl-terminated surface to form sensing layer, after blocked by BSA, CA19-9 can be detected at low concentration $10.0 \mathrm{U} / \mathrm{mL}$ with excellent reproducibility and acceptable specificity. Compared with conventional ECLIA, our assay is conducted direct detection of antigen without any label treatments, which has advantages of simple and short time. With the success of BIE system miniaturized, it can be applied to clinic.

\section{ACKNOWLEDGMENTS}

The authors gratefully acknowledge financial support from Ministry of Science and Technology of China (2008AA02Z419), Natural Science Foundation (10804083, 20845003) of China, Chinese Academy of Sciences (KJCX2.YW.M04), Key Laboratory of Microgravity, Institute of Mechanics, Chinese Academy of Sciences.

\section{REFERENCES}

\footnotetext{
${ }^{1}$ Arwin, H., and I. Lundstrgm. A reflectance method for quantification of immunological reactions on surfaces. Anal. Biochem. 145:106-112, 1985.

${ }^{2}$ Benters, R., C. M. Niemeyer, and D. Wöhrle. Dendrimeractivated solid supports for nucleic acid and protein microarrays. Chembiochem 2:686-694, 2001.

${ }^{3}$ Carpelan-Holmstrom, M., C. Haglund, P. Kuusela, H. Jarvinen, and P. Roberts. Preperative serum levels of CEA and CA242 in colorectal cancer. Br. J. Cancer 71: 868-872, 1995.

${ }^{4}$ Chen, Y., Y. Meng, and G. Jin. Optimization of off-null ellipsometry for air/solid interfaces. Appl. Opt. 46:84758481, 2007.

${ }^{5}$ Findlay, J. W. A., W. C. Smith, J. W. Lee, et al. Validation of immunoassays for bioanalysis: a pharmaceutical industry perspective. J. Pharm. Biomed. Anal. 21:1249-1273, 2000 .
} 
${ }^{6}$ Gürbüz, A. K., and A. M. Özel. Elevated carbohydrate antigen 19-9 levels in a patient with choledocholithiasis. Turk. J. Gastroenterol. 13:213-215, 2002.

${ }^{7}$ Hoimes, C. J., M. T. Moyer, and M. W. Saif. Biomarkers for early detection and screening in pancreatic cancer. J. Pancreas 10:352-356, 2009.

${ }^{8} \mathrm{Jin}$, G. Development of biosensor based on imaging ellipsometry. Phys. Stat. Sol. (a) 205:810-816, 2008.

${ }^{9}$ Jin, G., P. Tengvall, I. Lundström, and H. Arwin. A biosensor concept based on imaging ellipsometry for visualization of biomolecular interactions. Anal. Biochem. 232: 69-72, 1995.

${ }^{10}$ Jin, G., R. Jansson, I. Eundstrom, and H. Arwin. Imaging ellipsometry for biosensor applications. In: The 8th International Conference on Solid-State Sensors and Actuators and Eurosensors IX. Stockholm, Sweden, June 25-29, 1995, pp. 509-512.

${ }^{11}$ Jin, G., R. Jansson, and H. Arwina. Imaging ellipsometry revisited: developments for visualization of thin transparent layers on silicon substrates. Rev. Sci. Instrum. 67:2930 2936, 1996.

${ }^{12}$ Kausaite-Minkstimiene, A., A. Ramanaviciene, J. Kirlyte, and A. Ramanavicius. Comparative study of random and oriented antibody immobilization techniques on the binding capacity of immunosensor. Anal. Chem. 82:6401-6408, 2010.

${ }^{13}$ Kodadek, T. Protein microarrays: prospects and problems. Chem. Biol. 8:105-115, 2001.

${ }^{14}$ Lin, J., F. Yan, X. Hu, and H. Ju. Chemiluminescent immunosensor for CA19-9 based on antigen immobilization on a cross-linked chitosan membrane. J. Immunol. Methods 291:165-174, 2004.

${ }^{15}$ Luo, Y., and G. Jin. A compact imaging ellipsometer for label-free biosensor. In: 4th European Conference of the International Federation for Medical and Biological Engineering, edited by J. V. Sloten, P. Verdonck, M. Nyssen, and J. Haueisen. Antwerp, Belgium, 2008, pp. 1050-1052.

${ }^{16}$ Malati, T. Tumour markers: an overview. Indian J Clin. Biochem. 22:17-31, 2007.

${ }^{17}$ Nakagoe, T., T. Sawai, T. Tsuji, et al. Circulating sialyl Lewisx, sialyl Lewisa and sialyl Tn antigens in colorectal cancer patients: multivariate analysis of predictive factors for serum antigen levels. J. Gastroenterol. 36:166-172, 2001.

${ }^{18}$ Nygren, H., T. Sandström, and M. Stenberg. Direct visual detection of protein antigen: importance of surface concentration. J. Immunol. Methods 59:145-149, 1983.

${ }^{19}$ Qi, C., J.-Z. Duan, Z.-H. Wang, et al. Investigation of interaction between two neutralizing monoclonal antibodies and SARS virus using biosensor based on imaging ellipsometry. Biomed. Microdevices 8:247-253, 2006.

${ }^{20}$ Qi, C., W. Zhu, Y. Niu, et al. Detection of hepatitis B virus markers using a biosensor based on imaging ellipsometry. J. Viral Hepat. 16:822-832, 2009.

${ }^{21} \mathrm{Qi}, \mathrm{C}$., Y. Lin, J. Feng, et al. Phage M13KO7 detection with biosensor based on imaging ellipsometry and AFM microscopic confirmation. Virus Res. 140:79-84, 2009.

${ }^{22}$ Qi, C., X.-S. Tian, S. Chen, et al. Detection of avian influenza virus subtype $\mathrm{H} 5$ using a biosensor based on imaging ellipsometry. Biosens. Bioelectron. 25:1530-1534, 2010.

${ }^{23}$ Roh, H. W., N. R. Lee, Y. H. Cho, J. B. Jung, H. N. Chung, W. S. Yang, W. K. Lee, H. K. Lee, and G. H. Ryu. Development of a guideline for protein chip evaluation as medical devices. Curr. Appl. Phys. 6S1:261-265, 2006.

${ }^{24}$ Seurynck-Servoss, S. L., C. L. Baird, K. D. Rodland, and R. C. Zangar. Surface chemistries for antibody microarrays. Front. Biosci. 12:3956-3964, 2007.

${ }^{25}$ Sotorrío, P., A. Quirós, and J. M. Izquierdo. New immunoelectrochemiluminometric assay to measure thyrotropin. Clin. Chem. 43:2428-2430, 1997.

${ }^{26}$ Takahashi, Y., T. Takeuchi, J. Sakamoto, et al. The usefulness of CEA and/or CA19-9 in monitoring for recurrence in gastric cancer patients: a prospective clinical study. Gastric Cancer 6:142-145, 2003.

${ }^{27}$ Wang, Z. H., and G. Jin. A label-free multisensing immunosensor based on imaging ellipsometry. Anal. Chem. 75:6119-6123, 2003.

${ }^{28}$ Wang, Z.-H., and G. Jin. Covalent immobilization of proteins for the biosensor based on imaging ellipsometry. J. Immunol. Methods 285:237-243, 2004.

${ }^{29}$ Wang, Z.-H., Y.-H. Meng, P.-Q. Ying, C. Qi, and G. Jin. A label-free protein microfluidic array for parallel immunoassays. Electrophoresis 27:4078-4085, 2006.

${ }^{30}$ Wang, X., Y. Wang, H. Xu, H. Shan, and J. R. Lu. Dynamic adsorption of monoclonal antibody layers on hydrophilic silica surface: a combined study by spectroscopic ellipsometry and AFM. J. Colloid Interf. Sci. 323:18-25, 2008.

${ }^{31}$ Wang, C.-L., J.-Q. Li, H.-W. Li, et al. Soluble angiopoietin receptor Tie-2 in patients with acute myocardial infarction and its detection by optical protein-chip. Artif. Cells Blood Substit. Biotechnol. 37:183-186, 2009.

${ }^{32}$ Woollama, J. A., P. G. Snydera, and M. C. Rosta. Variable angle spectroscopic ellipsometry: a non-destructive characterization technique for ultrathin and multilayer materials. Thin Solid Films 166:317-323, 1988.

${ }^{33}$ Wu, J., H. Yu, Y. Shao, X. Han, and Y. Zhang. Significance of CEA and CA242 in the diagnosis of colorectal carcinoma. Chin. J. Cancer Res. 8:272-275, 1996.

${ }^{34} \mathrm{Wu}$, J., Z. Zhang, Z. Fu, and H. Ju. A disposable twothroughput electrochemical immunosensor chip for simultaneous multianalyte determination of tumor markers. Biosens. Bioelectron. 23:114-120, 2007.

${ }^{35}$ Xu, H., X. Zhao, C. Grant, J. R. Lu, D. E. Williams, and J. Penfold. Orientation of a monoclonal antibody adsorbed at the solid/solution interface: a combined study using atomic force microscopy and neutron reflectivity. Langmuir 22:6313-6320, 2006.

${ }^{36} \mathrm{Yu}$, L., C. M. Li, and Q. Zhou. Efficient probe immobilization on poly(dimethyl siloxane) for sensitive detection of protein. Front. Biosci. 10:2848-2855, 2005.

${ }^{37}$ Zhan, Q., and J. R. Leger. High resolution imaging ellipsometry. Appl. Opt. 41:4443-4450, 2002.

${ }^{38}$ Zhang, H.-G., C. Qi, Z.-H. Wang, G. Jin, and R.-J. Xiu. Evaluation of a new CA 15-3 protein assay method: optical protein-chip system for clinical application. Clin. Chem. 6:1038-1040, 2005. 